Gazi University
Journal of Science
http://dergipark.gov.tr/gujs

\title{
S-Band Active Phased-Array RF Beam Forming Networks
}

\author{
Ahmet HASTURK ${ }^{1}$ (D) Nursel AKCAM ${ }^{(10)}$, Funda Ergun YARDIM $^{2 *}$ \\ ${ }^{I}$ ASELSAN A.Ş. Karaoğlan Mahallesi, 06830 Gölbaşı,Ankara, TURKEY \\ ${ }^{2}$ Gazi University Faculty of Engineering Department of Electrical and Electronics Engineering Eti Mh. Yükseliş Sk. No: 5 Kat: 306570 \\ Maltepe/ANKARA, TURKEY
}

\author{
Highlights \\ - Performance of beamformers directly effect overall system performance. \\ - Multiple beam can be electronically directed at the same time in active phased array systems. \\ - In Active phased array systems, amplitude control of each antenna element can be done separately. \\ - True-time delay structures decrease gain losses due to beam squint.
}

\begin{tabular}{l} 
Article Info \\
Received: $05 / 09 / 2018$ \\
Accepted: $21 / 06 / 2019$ \\
Keywords \\
\hline Active phase-array \\
RF Beamforming \\
Electronically Scanned \\
True-time delay
\end{tabular}

True-time delay

\begin{abstract}
One of the most important Active Phased-Array networks is beamforming networks, which has electronically scanning feature. The performance of these networks directly effects the system's performance. By this reason, the design of these structures requires a serious labor and experience in order to design them in a simulation environment. In this paper, the design, implementation and measurement tests of a Radio Frequency (RF) beamforming network, which is necessary for active phased-array structures, are discussed. The performance of the designed beamforming card is examined with the measurements and tests of gain, phase shifter, True Time Delay (TTD), digitally controlled attenuator, and input-output return loss. The measurement results are compared with simulation for the designed beamforming card. Both measurement results and simulation were shown that the desired performance was provided for the designed beamforming card; however, it is more convenient to feed each antenna element with a single-channeled beamforming card. In this beamforming card, each channel's phase and amplitude can be controlled separately.
\end{abstract}

\section{INTRODUCTION}

In electronic warfare systems, a receiver structure is required to receive signal from threat and evaluate so as to perform the self-protection and, counter measure functions, and a transmitter structure, to jamming the threat. In order to perform these functions, receiver-transmitter structures need to have a beam, which is directed towards the location of a threat at the convenient power. Rapidly directing a beam and satisfying of a required power value are two important parameters for effective jamming. In order to create a beam with the traditional methods, a large-sized and mechanically controlled antenna and a high-power RF amplifier, to feed the antenna, are needed [1]. Because the beam direction can be mechanically controlled, time period, required to create a beam, is at the level of milliseconds. Furthermore, only one beam can be created at a time. The phased-array systems are developed to design structures, which can be created multiple beams at the same time; and can be directed electronically faster; and are smaller in size [2-3].

The phased-array structures are divided under two groups as passive phased-array and active phased-array ones, according to feeding level of the antenna elements (Figure 1). In the passive phased-array structures, all antenna elements are fed by one RF amplifier for the transmission units, while they are fed by singlechannel in the receiver structures. In the active phased-array structures, the amplitude control of each antenna element can be done separately. In order to feed this type of antenna, receiver and/or transmitter modules with lower power, compared to the passive phased-array structures are used [4-6]. 
In the phased-array structures, the beamforming is made in two ways as analog and digital. In the method of analog beamforming; the beam is directed by adjusting the required phase with using phase shifters. If instantaneous bandwidth is large for operating frequency band and the array is consisted of many units, TTD structures can also be used to decrease the gain losses due to the beam squint [7-8].
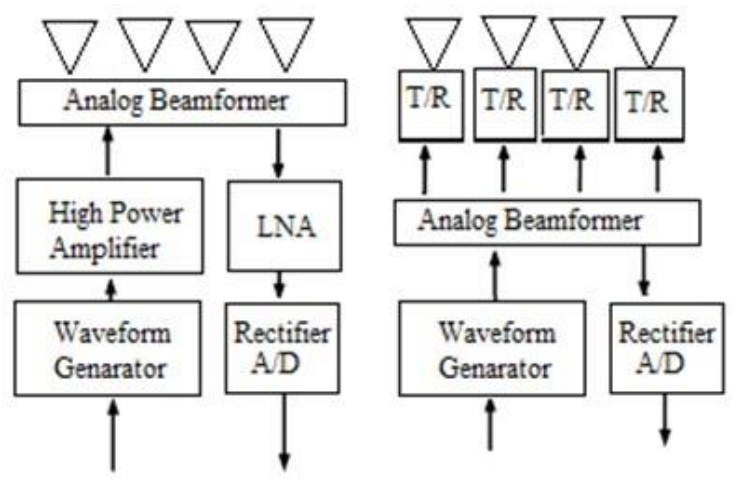

Figure 1. Passive-Active phased-array structures: LNA (Low Noise Amplifier), T/R (Transmitter/Receiver)

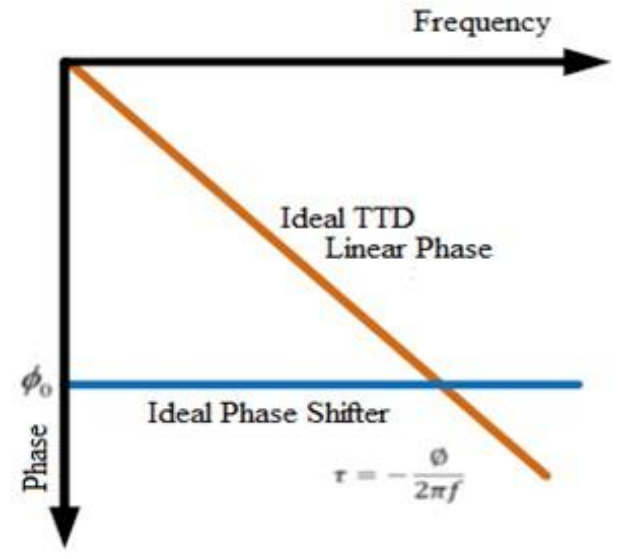

Figure 2. A Comparison of phase shifter and TTD

Phase response of phase shifter and TTD are shown in Figure 2. Unlike the phase shifter, the TTD has a negatively increasing phase performance. In figure $\tau$ is time delay, $\mathrm{f}$ is frequency and $\phi$ is phase [9-14].

In the digital beamforming method, a beam is directed after information on phase and time difference, which is required to direct the beam, is decreased to a level, which the analog-digital converter can process, by being numerically adjusted. In the digital beamforming structures [15], because a beam is directed on the basis of FPGA (Field Programmable Gate Array), it is an expensive method, compared to the analog beamforming method.

\section{MATERIAL AND METHODS}

\subsection{2-4 GHz Transmission Unit's Beamforming Structure}

In this paper, an analog beamformer design is realized such that one single board could be configured to work in transmit mode applicable for S-band $(2-4 \mathrm{GHz})$. The components are located on a printed circuit, according to the optimum gain and noise figures. In order to decrease the cost of the card designed in this study, a shared printed circuit design is made for the receiving and transmission structure. Therefore, the printed circuit card is used in receiving and transmission units by changing the directions or by-passing the components for two different configurations. 
In this study, an 8-channel beamforming card is designed and phase shifter and TTD structure, consisted of 3 cascaded stages, were used to direct the beam. Due to the size of the array and large instantaneous bandwidth, TTD structures are also used in addition to the phase shifters. In order to reduce the amplitude differences between the channels and decrease the level of the side lobes, digitally controlled attenuators are used. The materials, used in the designed beam direction card and its features are given in Table 1.

Table 1. Beamformer Card Strip Analysis

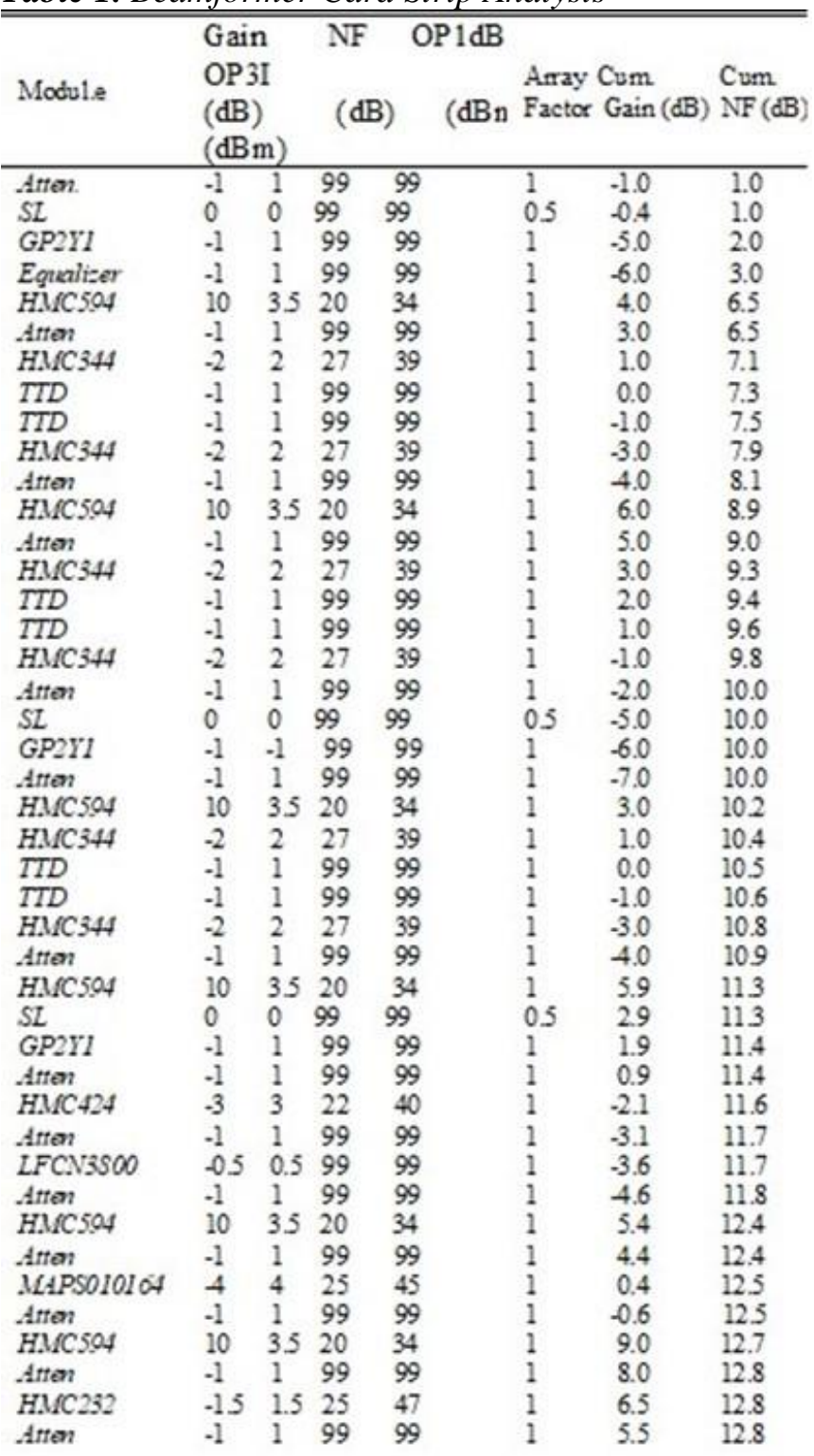

In Table I: $N F=$ Noise Figure, $O P 1 d B=$ Output ldB Saturation Point, $O P 31$ = Output Third Order Intersect Point, Cum = Cumvlative, SL = Splitting Loss, Atten. $=$ Attervi ation .

In this study, it was aimed that the VSWR (Voltage Standing Wave Ratio) value should be smaller than 1.9. In the measurements, RL (Return Loss) is measured instead of VSWR. VSWR return loss is given in (1) [16-17]:

$$
R L=20 \log _{10}\left|\frac{V S W R+1}{V S W R-1}\right| d B .
$$




\subsection{Card Performance - Measurement Results}

A beamforming network, with 8 channels, was designed in AWR simulation environment. The beamforming card block diagram, designed in this study, is shown in Figure 3. Due to many components on the card, a short circuit which is possible to occur in the reflow process, affects the other channels. Because every single channel combines through power divider structures, if RF performance of single channel decreases, it affects the general performance of the card -since all channels are interacting.

The measurement results of the gain of each channel, are seen in Figure 4. There was found a difference of $1.5 \mathrm{~dB}$ between the simulation and measurement results. The gain of each channel was calculated as $11 \mathrm{~dB}$ at the beginning and min. $8.1 \mathrm{~dB}$ at the end of the band from the simulation results. As from Figure 4 the measured gain is $9.5 \mathrm{~dB}$ at the beginning and min. $7.1 \mathrm{~dB}$ at the end of the band for 8 channels.

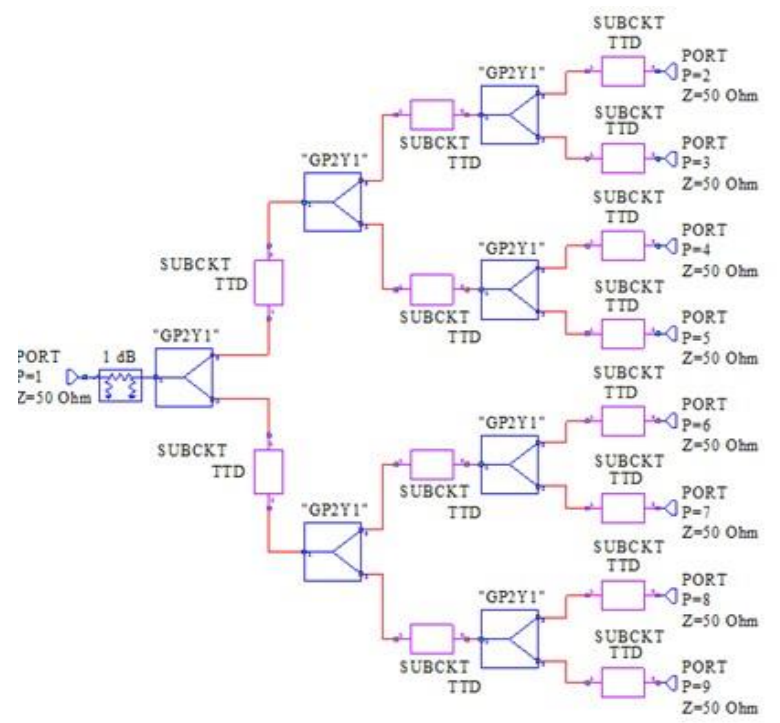

Figure 3. Beamforming card block diagram

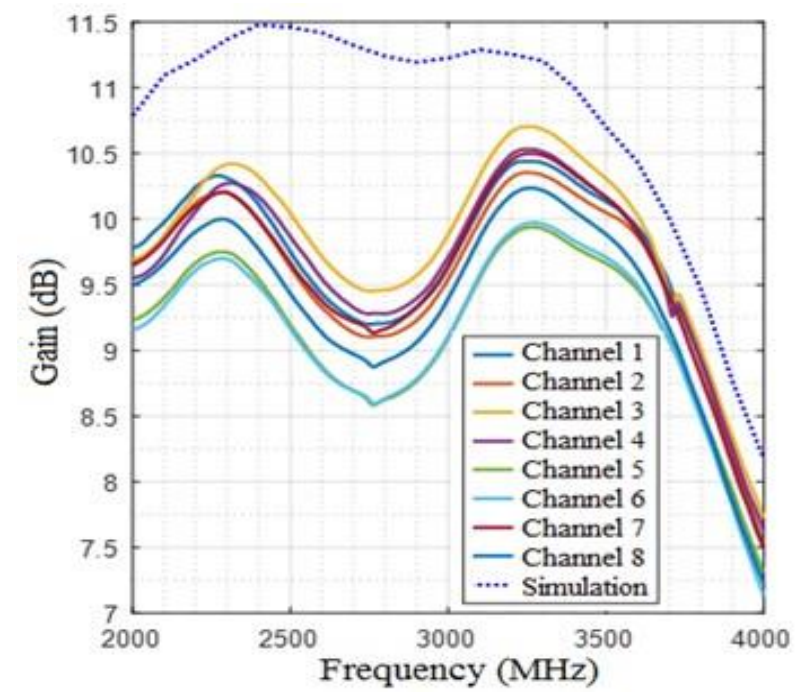

Figure 4. Gain measurements 
The differences between simulation results and measurements are related with the RF connector and DC block capacitors that are not included in the simulation. On the other hand, the amplitude change between the channels were measured as max. $1 \mathrm{~dB}$. This effect is caused from the tolerances of the elements used, from the process of PCB production and from the soldering of the components.
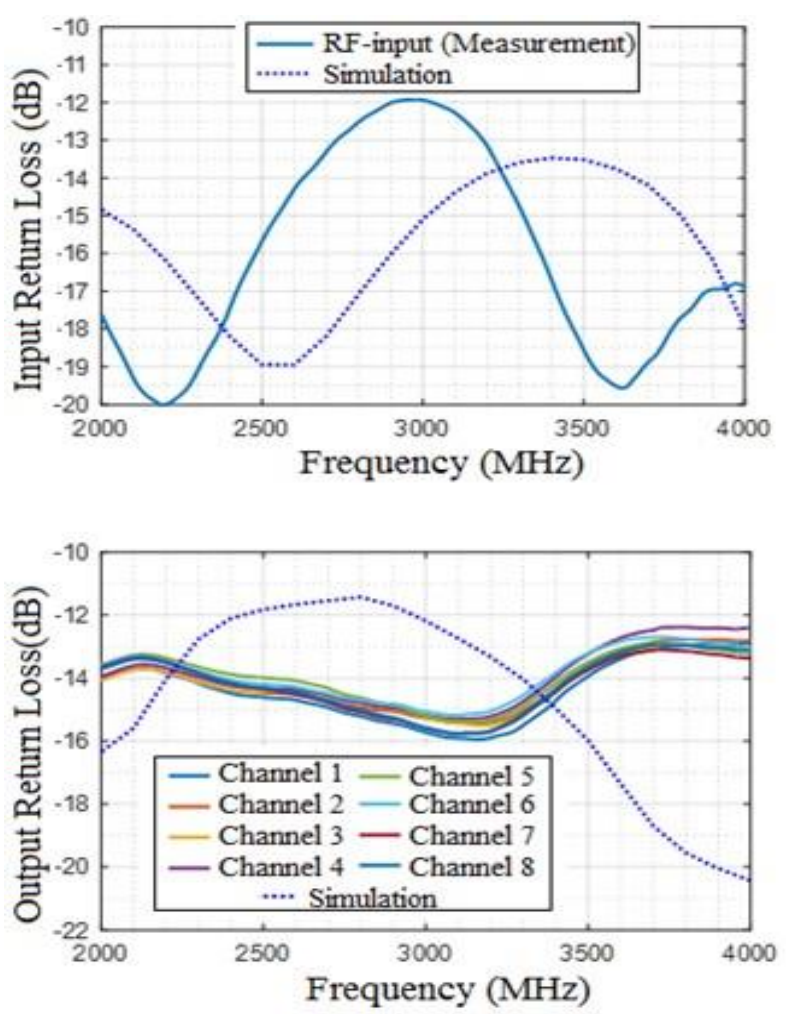

Figure 5. Input-Output return loss measurements

As it is seen in Figure 5, the return loss (input-output return losses) of input and output ports is measured as better than $12 \mathrm{~dB}$. The input and output return loss values (Figure 5) are less than $-10 \mathrm{~dB}$ in simulation and measurement results along the band. According to the measurement results, the input return loss value is measured as less than $-12 \mathrm{~dB}$, while the output return loss value is measured as less than $-13 \mathrm{~dB}$ for each channel. The difference in the figure is due to increasing parasitic effects and material tolerances at high frequencies. As the measured values are less than $-10 \mathrm{~dB}$ for each channel, the maximum power transfer and $50 \Omega$ line impedance across the card was satisfied.

It is seen in Figure 6 that 180 degrees bit of the performance of the designed card's phase shifter has $15^{\circ}$ phase errors. These phase errors are consisted of the characteristics of the phase shifter. By examining Figure 6 for the main bits of the phase shifter, the phase differences of 5.625, 11.25, 22.5, 45, 90 and 180 degrees were measured very close to the simulation results. The difference of 5 degrees between the simulation and the measurement result at the 180 degrees phase which gives the highest phase difference is within the material tolerances. When phase shifter measurements were taken, as the phase shifter used in each channel is the same, only the measurements of the first channel were reflected on the figure. 


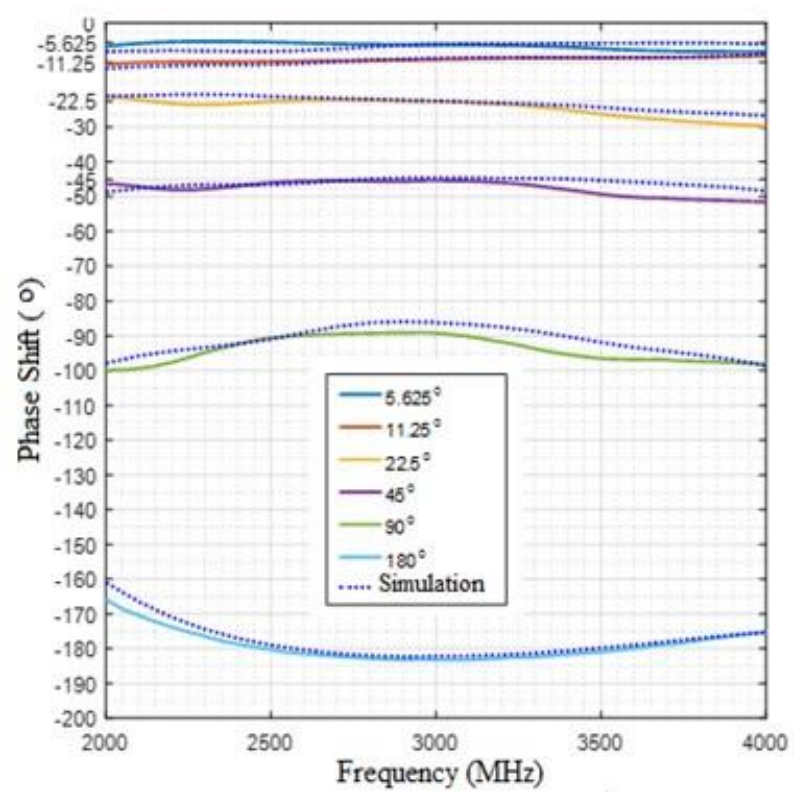

Figure 6. Phase shifter measurements

The measurement results of the digitally controlled attenuator show that the maximum amplitude error in the frequency band is $0.5 \mathrm{~dB}$ (Figure 7). If the measurements are examined, it can be seen that the digital attenuator provides $0.5 \mathrm{~dB}, 1 \mathrm{~dB}, 2 \mathrm{~dB}, 4 \mathrm{~dB}$ and $8 \mathrm{~dB}$ attenuation values along the frequency band (Figure 7).

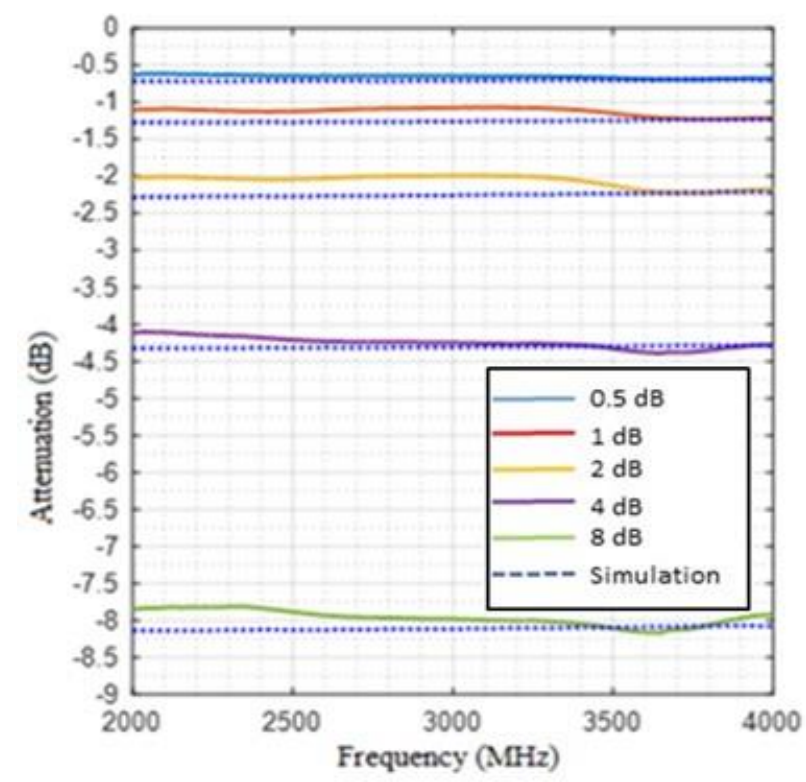

Figure 7. Attenuator measurements

A $0.5 \mathrm{~dB}$ difference was observed between the simulation and measurement results from the manufacturing tolerances of the digitally controlled attenuator. When the attenuator measurements were taken, as the digital attenuator used in each channel is the same, only the measurements of the first channel were reflected on the figure.

Figure 8 - Figure 10 show TTD measurements results, which are consisted of different stages, there is a true time delay error of $30 \mathrm{ps}$. 


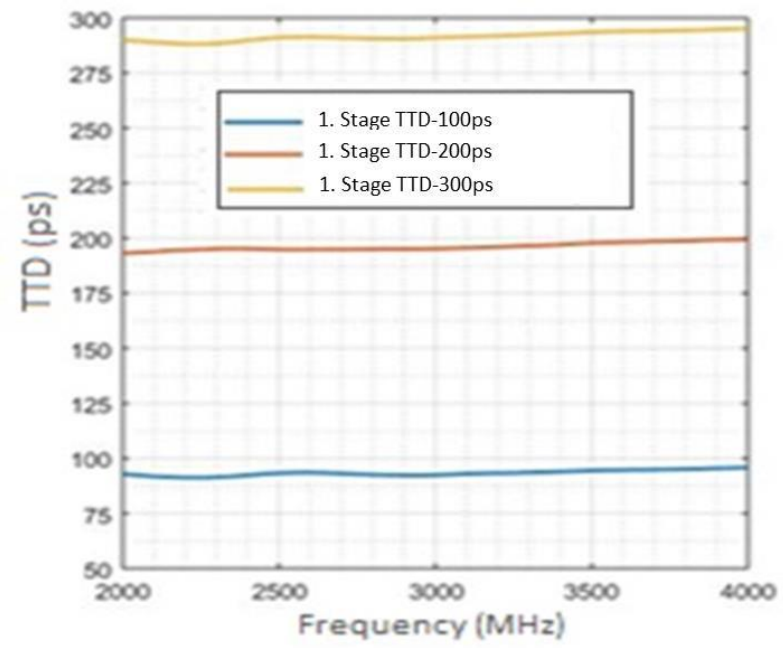

Figure 8. First stage TTD measurements

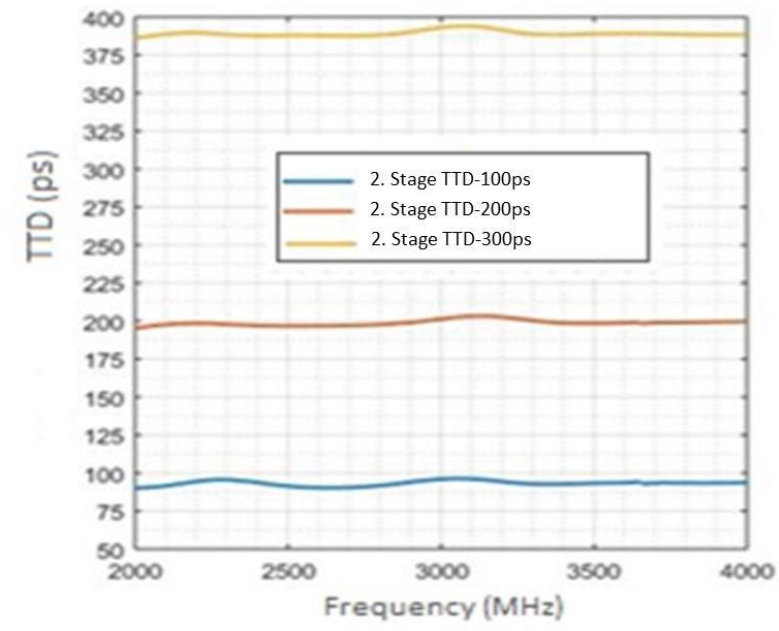

Figure 9. Second stage TTD measurements

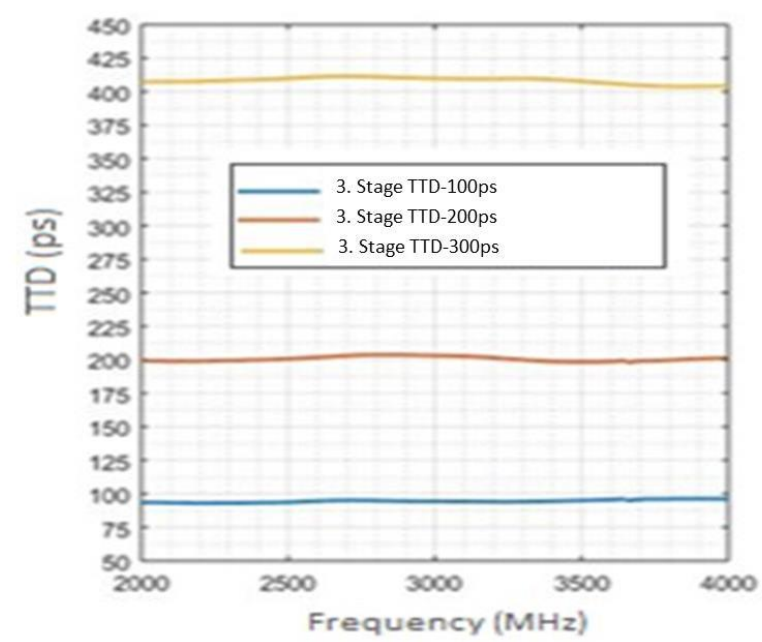

Figure 10. Third stage TTD measurements 
When first stage TTD measurements are examined, the desired 100, 200, 300 ps time delay steps are highly consistent with the simulation results with a maximum $10 \mathrm{ps}$ time difference (Figure 8). The 100, 200, 400 ps time delay steps expected for each block according to the last stage TTD (composed of two cascaded blocks) measurements overlap with a maximum of 15 ps difference compared to the simulated data (Figure 9 and Figure 10).

In a TTD structure consisting of three blocks in total, it is aimed to give time delay by connecting each stage with a SP4T switch structure. In the case of zero time delay, which is the reference channel is switched, the branches of the switches are connected directly to each other via the transmission line and measurements are made with reference to this channel. In this case, the other branches of the switch with different time delay material, must be that length of the reference branch, except for the total material used.

The main reasons for which some bits cannot give the expected delay precisely in measurements are coupling effects due to the proximity of switch branches arising from the extension or shortening of certain lines as well as the isolation of the SP4T switch when equal length lines are drawn due to the geometry of the switch and TTD material.

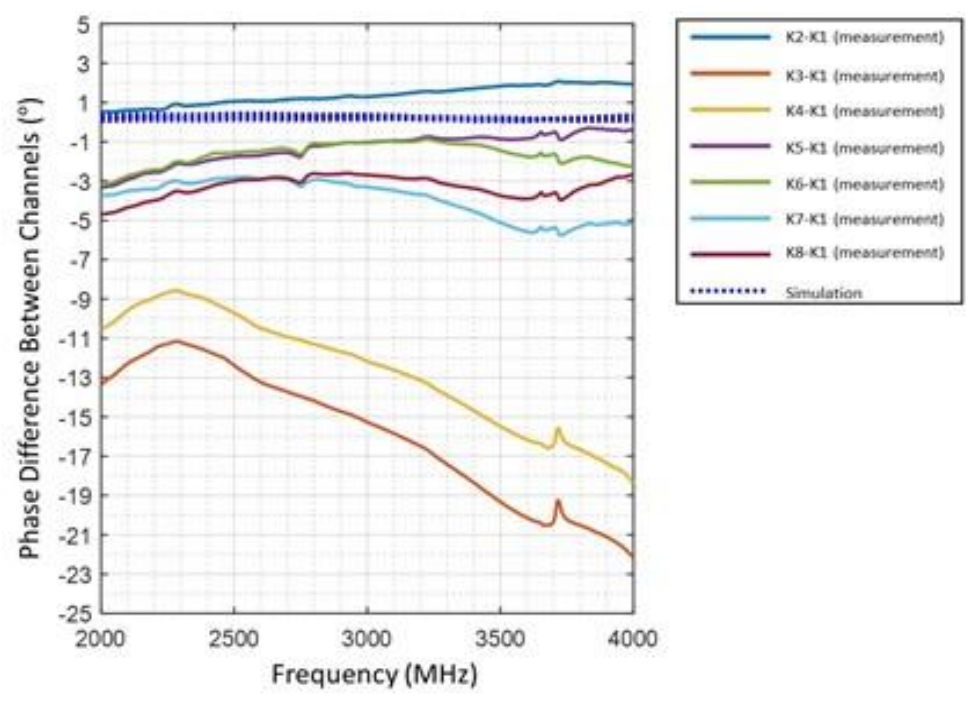

Figure 11. Phase difference between channels

When the phase difference between the channels are examined, it is expected that every channel will be equal to each other according to the results of simulation. In Figure11, near-20-degree phase differences have occurred at some channels at the end of the band. The phase differences between channels occurs when some lines cannot be drawn in equal length with very small tolerances due to material geometry and symmetry while the total line length of each channel is targeted to be equal to each other. In addition, when RF connectors and other components are soldered to the board, excessive or little soldering caused by improper contact of components with the card surface can directly affect phase difference.

\section{RESULTS AND DISCUSSION}

Phase array systems are preferred for military electronic warfare applications due to their advantages such as multi beam generation, electronical beam steering, and speed beam switching and small size. Since the beam is electronically directed in such systems, the beam steering structure is one of the most important blocks of the system design. For phase array beam steering structures, analog, digital and photonic-based beamforming approaches can be found in literature. Each approach has advantages and disadvantages for performance, cost and labor parameters.

In this paper, an analog beamforming board which can be used in active phase array systems consisting of 8 channels operating in S-band has been designed, manufactured and tested. The design consists of three 
different blocks; phase and amplitude adjustment block, first stage and final stage TTD blocks. In the first designed phase and amplitude adjustment block, there is found a digital phase shifter and a digital attenuator for using each channel. The phase and amplitude required for beamforming can be set independently for each channel by using this block. In the first stage TTD block, a switchable TTD structure is designed, which can be set jointly for each pair of channels and gives a maximum time delay of $300 \mathrm{ps}$ in total with 100 ps steps. In the final stage TTD block, a switchable TTD structure is designed with 2 cascade connections, each with a time delay of 400 ps with 100 ps steps each. By using the final stage TTD block, a maximum delay time of 800 ps can be set for common 4 channels. The design of the 8 -channel analog beamforming structure is completed by combining the beamforming card, designed in sub blocks, with the two-way power divider. In addition to phase shifters, it is aimed to reduce the gain reductions due to beam squint with the aid of TTDs added in sub-array design. In addition, with the help of the digital attenuators in each channel, the antenna elements were fed with different amplitudes and the side lobe was suppressed.

The printed circuit board is designed and manufactured for the card design completed on AWR. In the printed circuit design, it is aimed that each channel should have the same phase and the same amplitude by connecting the RF transmission lines with equal lengths.

\section{CONFLICTS OF INTEREST}

No conflict of interest was declared by the authors.

\section{REFERENCES}

[1] Yasser, A.R., “Active phased array radar system”, Lockheed Martin, (2008).

[2] Parker, D. and Zimmermann, D.C., "Phased arrays-Part I: theory and architectures", IEEE Transactions on Microwave Theory and Techniques, 50(3): 678-687, (2002).

[3] Yoon, M. and Nam, S., "Avoidance of off-switch Resonance in True Time Delay Line using Cascaded Switches", in URSI Asia-Pacific Radio Science Conference, Seoul, South Korea, (2016).

[4] Visser, H.J., “Array and Phased Array Antenna Basics”, Wiley, (2005).

[5] Hansen, R C., "Phase array antenns" Second Edition, Wiley, (2009).

[6] Mailloux, R. J., "Phase array antenna handbook" Second Edition, Artech House, (2005).

[7] Montebugnoli, S., Bianchi, G., Cattani, A., Ghelfi, F., Maccaferri, A. and Perini, F., "Some notes on beamforming", The Medicina IRA-SKA Engineering Group, (2004).

[8] Lee, J. J., "RF photonics for beamforming and array applications", in Optics Microwave Interactions. NATO Research and Technology Organization, paper 4, publication RTO-EN-028, 4.1-4.9, (2002).

[9] Longbrake, M., Buck, J., Buxa, P., Dalrymple, T., McCann, J., Neidhard, R., Watson, P., Zeller, K., Garber, B. and Kuhl, D., "TELA testbed time delay beam steering demonstration", Antenna Measurement Techniques Association Symposium, Salt Lake City, Utah, USA, (2009).

[10] Pulikkaseril, C., Li, L., Yi, X. and Poole, S., "The use of uncooled DFB lasers in microwave photonic phase shifters", in Opto-Electronics and Communications Conference (OECC), Shanghai, China, (2015).

[11] Frankel, M. Y., Esman, R. D. and Parent, M. G., "Array transmitter/receiver controlled by a true timedelay fiber-optic beamformer”, IEEE Photon. Technol. Letter, 7(10):1216, (1995). 
[12] Liang, Y., Domier, C. W. and Luhmann, N. C., "MEMS based true time delay technology for phased antenna array systems", Asia-Pacific Microwave Conference, Bangkok, Thailand, (2007).

[13] Kim, M. Hacker, J. B., Mihailovich, R. E. and DeNatale, J. F., "A DC to-40 GHz four-bit RF MEMS true-time delay network", IEEE Microwave and Wireless Components Letters, 11(2):56-58, (2001).

[14] Temir, K., "True-Time Delay Structures for Microwave Beamforming Networks in S-Band Phased Arrays", METU, (2013).

[15] Haynes, T., "Primer on digital beamforming”, Spectrum Signal Processing, 1-15, (1998).

[16] Trevor, S. B., "Definition and misuse of return loss", IEEE Antennas \& Propagation Magazine, 51(2): 166-167, (2009).

[17] Skolnik, M. I., "Introduction to radar systems", 3rd edition New York, NY: McGraw-Hill, (2001). 